\title{
Transgender Research in the 21st Century: A Selective Critical Review From a Neurocognitive Perspective
}

\author{
Sven C. Mueller, Ph.D., Griet De Cuypere, M.D., Ph.D., Guy T'Sjoen, M.D.
}

Gender dysphoria describes the psychological distress caused by identifying with the sex opposite to the one assigned at birth. In recent years, much progress has been made in characterizing the needs of transgender persons wishing to transition to their preferred gender, thus helping to optimize care. This critical review of the literature examines their common mental health issues, several individual risk factors for psychiatric comorbidity, and current research on the underlying neurobiology. Prevalence rates of persons identifying as transgender and seeking help with transition have been rising steeply since 2000 across Western countries; the current U.S. estimate is $0.6 \%$. Anxiety and depression are frequently observed both before and after transition, although there is some decrease afterward. Recent research has identified autistic traits in some transgender persons.

Identifying with the sex opposite to the one assigned at birth often evokes serious distress and dysphoria, which are likely to be exacerbated by the legal, social, vocational, family, and relationship consequences of such an identification, including social stigma. Moreover, such distress can lead to a variety of mental health problems. As a result, many individuals experiencing gender dysphoria search for gender-transition-related care (1). Our aim in this selective review is to provide an overview of current research in transgender persons, focusing on mental health and mental health problems and their neurobiological and neurocognitive and affective underpinnings. Currently, to facilitate transition, lines of care include cross-sex hormone treatment (CSHT) and/or gender-affirming surgery. The beneficial effects of these lines of care on mental health and neurobiology will be scrutinized here.

Transgender care is complex and highly interdisciplinary. It involves a variety of health professionals, including endocrinologists, gynecologists, urologists, surgeons, voice and communication specialists and therapists, dermatologists, and various providers of mental health services (psychiatrists, clinical psychologists, counselors, etc.) (2). In line with the goals of the World Professional Association for Transgender Health (2) to de-psychopathologize being transgender, the terminology has been constantly evolving in recent years. For persons identifying with the sex opposite to the one assigned at birth, we use the currently preferred
Forty percent of transgender persons endorse suicidality, and the rate of self-injurious behavior and suicide are markedly higher than in the general population. Individual factors contributing to mental health in transgender persons include community attitudes, societal acceptance, and posttransition physical attractiveness. Neurobiologically, whereas structural MRI data are thus far inconsistent, functional MRI evidence in trans persons suggests changes in some brain areas concerned with olfaction and voice perception consistent with sexual identification, but here too, a definitive picture has yet to emerge. Mental health clinicians, together with other health specialists, have an increasing role in the assessment and treatment of gender dysphoria in transgender individuals.

Am J Psychiatry 2017; 174:1155-1162; doi: 10.1176/appi.ajp.2017.17060626

terms "trans persons" or "transgender persons" throughout this review. The term "transgender men" is used for persons transitioning from female to male and "transgender women" for persons transitioning from male to female. In most cases, but not all, we refer to persons with gender dysphoria visiting mental health services and seeking gender-affirming surgery. Whereas some individuals may not be distressed by experiencing incongruence between their gender identity and their sex assigned at birth, a formal DSM-5 diagnosis of gender dysphoria is still necessary in many countries to facilitate access to mental health care and other professional care that will help in the transition to the experienced gender. The term "cisgender persons" is used throughout the review to describe persons who did not transition and who identify with their sex assigned at birth, for example, a female identifying as female and with female sex assigned at birth.

\section{EPIDEMIOLOGY}

Epidemiologically, true prevalence rates of transgenderism are difficult to establish because of sampling biases (2). Concerns in establishing prevalence rates start with the criteria for inclusion, that is, whether the person has already undergone gender-affirming surgery, is receiving CSHT, or has presented with gender dysphoria to a mental health provider. Because of this bias, estimated prevalence rates 
have traditionally been underestimates, as some gendervariant individuals (i.e., associating oneself with a sex different from the one assigned at birth without being dysphoric) may never present to a mental health care provider. To overcome these limitations, recently published prevalence estimates are conducted using meta-analytic methods or population-based surveys. Using a meta-analysis on reported prevalence rates, Arcelus et al. (3) estimate the prevalence of persons being transgender at around 4.6 per 100,000, with 6.8 for transgender women and 2.6 for transgender men. Exploiting nationally available population surveys in the United States, Flores et al. (4) suggest a total prevalence of $0.6 \%$ for adults in the U.S. population identifying as transgender persons. The prevalence of gender dysphoria has been increasing worldwide in the past two decades. Potential reasons could be that concerned persons feel freer to disclose their dysphoria and to seek help because of more openness in society, changes in legislation, and a trans-affirmative approach on the part of mental health and medical care providers, the media, and social media. However, other changes in inter- or intraindividual factors are also plausible.

Of note, genetic issues in the synthesis of steroid hormones do not appear to contribute to the prevalence of gender dysphoria. Individuals with disorders of sexual development, such as congenital adrenal hyperplasia, complete or partial androgen insensitivity syndrome, or other, more rare variants of disorders of sexual development, do not meet the diagnostic thresholds for gender dysphoria $(5,6)$. Rather, it has been reported that women with congenital adrenal hyperplasia may be more likely to have a non-heterosexual orientation $(7,8)$.

\section{MENTAL HEALTH IN TRANSGENDER PERSONS}

Much cross-sectional work on mental health in transgender persons has focused on documenting the psychological or psychiatric problems that may affect a transgender person. In a large initiative across four European countries, Heylens et al. (9) reported that $38 \%$ of transgender persons with gender identity disorder $(\mathrm{N}=305)$ currently had axis I diagnoses, the majority of which were affective (27\%) and anxiety disorders (17\%). Notably, lifetime prevalences of axis I diagnosis, affective problems, and anxiety were estimated at $70 \%, 60 \%$, and $28 \%$, respectively, while axis II diagnoses were estimated at $15 \%$. Substance use disorders, eating disorders, and psychotic disorders were less prevalent, at $16 \%, 2 \%, 1 \%$, respectively. The problematic high rates of psychological and/or psychiatric problems in trans persons remained even when participants were matched with cisgender comparison subjects and additional factors such as age, natal sex, and new assigned sex were taken into account in a retrospective Swedish cohort study (10). While such high rates of psychopathology are consistently reported in the literature, there is disagreement regarding sex specificity. Whereas some studies document higher prevalences of psychopathology in transgender women relative to transgender men (11-13), one study reported the reverse (14).
Interestingly, in addition to the axis I disorders mentioned above, attention in the field has recently shifted to the presence of autism spectrum disorders or autistic traits in transgender persons. While any conclusions would still be premature, currently available data appear to suggest higher rates of cooccurring autistic traits in trans persons with gender dysphoria relative to the general population in Dutch children $(\mathrm{N}=204)$ (15) and British adults (16). In the latter study (16), trans men $(\mathrm{N}=61)$ but not trans women $(\mathrm{N}=198)$ had higher autism quotient scores than cisgender men and women but lower scores than 125 patients with a diagnosis of Asperger's syndrome. These data suggest that while autistic traits in trans persons may be higher than in the general population, they may be lower than in the population of people on the autism spectrum and are dependent on sexual orientation or sex.

Despite these concerning rates of psychopathology and axis I and II disorders, emergent longitudinal research has begun to document the positive change in mental health associated with successful transition. This research shows significant reductions in psychopathology after transition, down to a level resembling normative data (for a comprehensive review, see reference 17). In one longitudinal study of 107 transgender persons (18), symptoms of anxiety, depression, and psychoticism and measures of global severity and functional impairment were all significantly reduced after 12 months of CSHT. Similarly, another study (19) also tested the beneficial effects of CSHT on mental health in both a crosssectional and longitudinal design. Cross-sectionally, the study found that CSHT alleviated depressive symptoms in trans men but not trans women relative to trans persons not receiving hormones. With hormone therapy, levels of body uneasiness in both genders also diminished. Crucially, longitudinally across four time points (at 3, 6, 12, and 24 months of follow-up), CSHT was associated with significant reductions in psychopathology (on the Symptom Checklist-90 global severity index), depressive symptoms, body uneasiness levels, and gender dysphoria in both genders (19). In sum, while being trans may be associated with increased mental health problems, predominantly affective disorders, promising findings are emerging that indicate a reduction of mental health-related psychopathology, including depression and body dissatisfaction, with time and hormonal treatment.

Nonetheless, a major concern in trans persons remains the risk of suicidality (suicidal thoughts, suicide attempts, and suicide rates) as well as nonsuicidal self-injury, such as cutting, hitting, or burning oneself. These behaviors may reflect a maladaptive way to regulate one's emotion, or selfpunishment in response to external or internal pressure and social stigma. Because of these high-risk behaviors, the past decade has seen a surge in studies assessing the presence of suicidality and nonsuicidal self-injury in trans persons, with steadily increasing sample sizes. Rates of lifetime suicidality and suicide attempts for trans persons are alarming, ranging from $30 \%$ to $81 \%$ (the prevalence rate of suicide attempts in the overall U.S. population is estimated at 4.6\%) $(9,20-22)$. Significant predictors of suicide include a past history of maltreatment, gender victimization, depression, substance 
abuse, and young age (20-22). Rates for lifetime nonsuicidal self-injury in the pretransition period are estimated at $38 \%$ for the total trans population, with $57.7 \%$ in trans men and $26.2 \%$ in trans women (11). Although another study reported much lower rates of nonsuicidal self-injury (19\%) (23), both studies found that trans men were more at risk of nonsuicidal self-injury than trans women. These results are shared by investigations in pediatric populations, which also report elevated rates of self-harm in children and adolescents with gender dysphoria (24), suggesting that suicidality and self-injurious behavior are prominent across the lifespan in this population.

Long-term data assessing suicidality and nonsuicidal selfinjury after transition are fewer but continue to signal cause for concern. Retrospective data on 1,331 trans persons who visited a university gender clinic (25), with a median follow-up of 18.5 years, indicated high mortality rates among trans women, with a total mortality $51 \%$ above the general population average (rates of total and cause-specific mortality among trans men did not differ significantly from those of the general population). Causes of death included heart and lung problems, neoplasm, HIV/AIDS, and suicide (17 trans women relative to one trans man). Death by suicide and suicide attempts after transition were equally alarming in the Swedish cohort study by Dhejne et al. (10), who reported an incidence of 2.7 per 1,000 person-years for death by suicide and 7.9 for suicide attempts. Notably, the National Transgender Discrimination Survey uncovered a high prevalence of $41 \%$ for suicide attempts in trans persons-double that reported in the LGB (lesbian, gay, bisexual) community, which is estimated at $10 \%-20 \%$ for the U.S. population (26). Such high rates of suicide risk in trans persons, even after transition, would mandate that trans persons presenting at the clinic not only be assessed for self-injurious behavior but also be monitored longitudinally.

Indeed, the recognition of being trans as an identity, rather than as a mental health disorder (2), is shifting the focus of the clinician's preassigned role. Given the high rates of mood and affective disorders as well as high suicidality in trans persons, especially before transition, clinicians must be able to diagnose not only gender dysphoria but also interrelated psychological and/or psychiatric problems as well as psychological problems that may masquerade as gender dysphoria. In addition, it is recommended that mental health care providers be familiar with the criteria for diagnosis and treatment of gender dysphoria and be able to care for the patient during the entire transition process and afterward.

\section{SOCIAL, ENVIRONMENTAL, AND INDIVIDUAL FACTORS}

Social, legal, vocational, and environmental factors contribute greatly to the well-being of trans persons. Whereas the level of life satisfaction among 6,771 trans persons in a large European Union lesbian, gay, bisexual, and transgender (LGBT) survey $(\mathrm{N}=93,079)$ was similar to that of the general population, it was nonetheless somewhat lower than that of nontrans respondents from the LGB community (27). This report also uncovered continuing problems that trans persons face with stigma, harassment, and violence and maltreatment in their personal and professional lives. Forty-six percent of trans persons in the survey reported having felt discriminated against or harassed within a 12-month period because of being perceived as trans (27), including $30 \%$ of trans persons when looking for a job and $23 \%$ at the workplace. Such social and environmental factors very likely contribute to mental health problems and suicidality. In a sample of U.S. LGBT youth ( $\mathrm{N}=246$, ages $16-20)$ (28), prospective LGBT victimization and low social support were associated with suicidal ideation, and self-harm was associated with (among other factors) childhood gender nonconformity, prospective hopelessness, and victimization.

Interindividual factors, including sexual orientation and physical appearance, may also contribute to well-being and mental health. In an interesting investigation into putative subtypes within the transgender population, Smith et al. (29) gathered data from 187 trans persons who had completed gender-affirming surgery and examined the role of sexual orientation on personality variables, physical appearance, onset of gender dysphoria (documented gender identity disorder), and psychopathology. Participant's physical appearance (facial hair, height, figure, nose, muscularity, speech, etc.) was rated in terms of how compatible this appearance was with the new (preferred) sex. These ratings showed that androphilic trans men compared with gynephilic trans women had a higher matching physical appearance. Moreover, androphilic trans women and gynephilic trans men, compared with gynephilic trans women and androphilic trans men, reported more gender identity disorder symptoms in childhood. Similarly, trans men also reported more gender identity disorder symptoms at early ages relative to trans women and applied for transition at an earlier age. These results may point to different underlying factors and motivations. Smith et al. (29) suggest that whereas androphilic trans women may have a strong aversion to their sex organs, motivating earlier transition, gynephilic trans women may have grown up as masculine boys and may also have entered marriage and fathered children, thus leading to later transition. Similarly, androphilic trans men reported more psychological problems than gynephilic trans men, suggesting that a combination of sexual attraction to a specific gender, physical appearance, and societal expectations may exert different effects on individuals with different sexual orientations or different age at onset of gender dysphoria (30).

The data are currently ambiguous, however, as an Italian study (19) found that although subjective gender dysphoria, depressive symptoms, and psychopathology decreased with CSHT, the social and socio-legal factors of gender dysphoria increased, particularly for trans men. These authors argued for a culturally specific explanation of this effect but regrettably did not explore this option further. However, the findings from the European Union survey cited above (27) 
support such conjectures, given substantial differences among member states in perception of discrimination, social stigma, and hate-related crime. However, more converging evidence and research endeavor in this domain are needed before more definitive conclusions can be drawn. In addition to research into these social and environmental factors, much recent work has examined the underlying neurobiology in transgender persons.

\section{STRUCTURAL NEUROANATOMY AND RESTING-STATE ACTIVITY}

Despite intensive searching, no clear neurobiological marker or "cause" of being transgender has been identified. Yet functional MRI (fMRI) and structural MRI studies of the brain in trans persons have been surging over the past decade to chart the complex contributions of underlying neurobiology. Hunting for a neurobiological etiology in trans persons, the majority of studies in this domain have examined whether the brains of trans persons resemble those of their sex assigned at birth, resemble those of their gender identity, or are intermediate to either sex. These structural MRI studies have predominantly focused on persons pretreatment and have reported mixed findings. Various factors contribute to these mixed results, including the use of different regions of interest, as well as statistical power issues, with sample sizes ranging from single case studies to around 24 participants per group, with slightly higher samples for cisgender control groups (31). In some of the largest studies available in hormonally untreated transgender persons, gray matter volume $(32,33)$ and total brain volume $(33)$ were similar for trans persons relative to their sex assigned at birth. Findings in other global measures, such as in the corpus callosum, are mixed and do not show any difference in size in trans persons before (34) or after CSHT (35), although they indicate a corpus callosum shape consistent with their gender identity (36). As for subcortical structures, putamen volume is either larger (32) or smaller (33) in trans women relative to cisgender men, with other studies suggesting a larger volume in trans women relative to cisgender women (35). Coming close to providing a neurostructural correlate of being a trans person, a valuable histological study in 42 postmortem brains (37) reported that the size of the hypothalamic uncinate nucleus (INAH-3) in 10 trans women resembled that in cisgender women, that is, it was consistent with their gender identity rather than their sex assigned at birth. Even though the trans persons in this postmortem study had been hormonally treated, the lack of testosterone did not appear to be the primary causative factor, given the absence of such an anatomical effect in nontrans castrated men, five of whom were also examined in the study. These data highlight the importance of paying close attention to regionally specific, minute structures when assessing which brain areas conform to gender identity rather than sex assigned at birth.

In comparison to these studies on gray matter volume, the few available white matter studies agree on an intermediate phenotype for trans persons in many sexually dimorphic fascicles yet disagree on the parameters tested and the precise order of group findings. In 3-T diffusion tensor imaging studies, Kranz et al. (38) documented widespread group differences in mean diffusivity between four groups (hormonally untreated transgender men and women and cisgender men and women). Cisgender women had the highest mean diffusivity values, followed by transgender men, transgender women, and cisgender men. Moreover, no differences were found in fractional anisotropy. Directly contrasting these findings, Rametti et al. documented, in separate reports on trans men (39) and trans women (40), differences in fractional anisotropy, with larger values for cisgender men relative to cisgender women, values for trans men being more similar to cisgender men, and values for trans women falling between those of the cisgender groups.

Although markedly fewer longitudinal studies or studies in trans persons after gender-affirming surgery and/or CSHT have been published, the few that have are promising. Confirming histological findings of a female-sized hypothalamus in trans women (37), Hulshoff Pol et al. (41) documented a decrease in hypothalamus size in this cohort with CSHT. Conversely, Kim et al. (42) found an increase in hypothalamic volume in trans men, suggesting plasticity and sensitivity of the hypothalamus to CSHT to shift toward the identified sex. A treatment study examining cortical thickness after 6 months of CSHT (43) indicated a variety of cortical thickness increases with androgen treatment in trans men and decreases with estrogens and antiandrogens in trans women. Again providing complementary evidence, while that study reported a volume increase in the global ventricular system in trans women, other groups have documented specific reductions in third ventricle size in trans men with hormonal treatment $(35,41)$. Some, however, have suggested that this finding is related to volumetric changes in adjacent gray matter structures, including the hypothalamus (41), a conjecture requiring further confirmation. However, serious caveats of currently available structural MRI studies include small numbers of transgender persons and cisgender comparison subjects (41), an absence of a cisgender comparison group (to establish baseline values for cisgender men and women) (43), an absence of pretreatment data (35), or separate publication of trans men and trans women, thus not allowing direct comparison $(39,40)$. Moreover, given the clinical implications of early versus late age at onset of gender dysphoria and sexual orientation (30), such variables deserve future scrutiny.

In addition to characterizing gray and white matter differences in transgender persons, structural and functional connectivity studies have also begun to emerge $(44,45)$. In a structural connectivity study of transgender persons before CSHT, Hahn et al. (44) documented decreased structural hemispheric connectivity ratios for transgender persons relative to cisgender persons in subcortical brain areas. In an MRI study examining local resting-state activity, Mueller et al. (45) recently observed that circulating androgen levels 
in trans men were associated with local resting-state activity in the frontal cortex and the cerebellum, an effect that was moreover linked to CSHT duration, at least in the cerebellum. No such effects of estrogen were found in transgender women. Taken together, structural MRI studies appear to show, depending on the brain region queried, an intermediate phenotype or a phenotype consistent with gender identity in trans persons that may shift further toward the experienced gender with CSHT (with other brain areas being consistent with sex assigned at birth). Yet, a unique neurobiological locus of "being transgender" remains to be identified, and more corroborative evidence utilizing larger and betterpowered controlled studies is needed before any definitive conclusions can be drawn. Joint imaging initiatives to compensate for these shortcomings are lacking at this stage. Nonetheless, some of these structural discoveries have led to important work on the functional implications of these changes.

\section{FUNCTIONAL NEUROANATOMY}

Although currently even more limited than structural imaging studies, fMRI studies on neurocognitive or affective processes have emerged, creating an intriguing line of work based on earlier anatomical and histological findings. Indeed, exploiting the early discovery of a female-like hypothalamus (37) and bed nuclei of the stria terminalis (BNST) (46) in transgender women, researchers recently aimed to probe the functional implications of this finding. The hypothalamus and BNST are centrally connected with other limbic structures such as the amygdala and the hippocampus and occupy a central role in hormonal signaling in sex-differentiated behavior. Studies set out to assess this impact in olfactory processing. Males and females are sensitive to estratetraenol and androstadienone, respectively, both steroid compounds with pheromone capability in humans and known to activate the hypothalamus. Berglund et al. (47) discovered that activation of the hypothalamic network to olfactory encounter of androstadienone in trans women was similar to that of cisgender women and different from that of cisgender men. Burke et al. (48) replicated this finding with 3-T fMRI in a larger sample of either prepubertal or pubertal children and adolescents with gender dysphoria (36 children and 38 adolescents) and without gender dysphoria (39 children and 41 adolescents). Interestingly, these additional developmental findings revealed that the sexually differentiated response of the hypothalamus was already present when comparing prepubertal cisgender boys with cisgender girls but that the difference in youths with gender dysphoria only began to emerge in adolescence and was consistent prepubertally with sex assigned at birth.

Whereas olfaction is a largely subliminal process, voice and speech perception are both processed sub- and supraliminally and contribute much to the perception of gender. Therefore, attention in transgender care is also directed toward the perceived voice and speech before and after transition. In transgender men, testosterone treatment may deepen the voice to approach male vocal pitch. However, in transgender women, estrogens do not have such an effect and vocal surgery and/or speech therapy may be needed (2). Indeed, speech therapists, speech-language pathologists, or speech-voice clinicians may all contribute to helping a transgender person accomplish the pitch, intonation, speech rate, or phrasing patterns as well as the nonverbal communication of their desired sex (2). Related fMRI research has examined how a male or female voice is perceived in trans women. Junger et al. (49) examined voice gender perception of male and female voices in a German sample of hormonally treated $(\mathrm{N}=16)$ and untreated $(\mathrm{N}=17)$ trans women in comparison to cisgender men $(\mathrm{N}=21)$ and women $(\mathrm{N}=20)$. These male or female voices were presented "pure" or were modified and presented in semitone steps toward one or the other gender. Whereas cisgender men differed from cisgender women in their response times when identifying voices of the opposite sex, reaction times were in between for transgender women. Consistent with such a response pattern, when voices were morphed toward the other sex, neural activation in the superior frontal gyrus in trans women was more similar to that of cisgender women and less similar when compared to cisgender men.

Other neurocognitive work in transgender persons has focused on functions commonly hypothesized to show "typical" sex differences in performance and to assess the extent to which CSHT would shift performance toward the gender identity in trans persons. Two such sex-stereotypical tasks are mental rotation (a spatial cognition task, said to favor males) and language and verbal fluency tasks (said to favor females). Starting with the first of these, in the largest crosssectional study to date on mental rotation abilities in trans persons, hormonally treated trans women $(\mathrm{N}=18)$ exhibited reduced parietal lobe activation, a region commonly active during mental rotation, relative to cisgender men but increased activation in the right orbital and dorsolateral prefrontal cortex relative to cisgender women (50). By contrast, no group effects were observed for trans men. Given the absence of pretreatment data in that study, it is difficult to assess whether the observed effects resulted from the CSHT or existed prior to it. Interestingly, a partial answer to this question is provided by Schöning et al. (51), who documented increased parietal cortex activation during mental rotation in cisgender men relative to both hormonally treated and untreated trans women. This would suggest that transgender women are more similar to their gender identity in their neural response during mental rotation, an effect that may occur prior to hormonal treatment.

In tasks hypothesized to favor females, findings have been mixed. In a pretreatment sample of adolescents with gender dysphoria, Soleman et al. (52) reported better behavioral performance in trans girls in a verbal fluency task (i.e., word production). Curiously, these trans girls resembled neither their sex assigned at birth nor their gender identity but produced more words (phonetic fluency) than any of the 
FIGURE 1. Overview of Key Issues and Findings in Current Transgender Research

\section{Key findings}

- Prevalence of number of people presenting to gender clinics is increasing

- Evidence of biological etiology is still limited

- Trans persons experience a high rate of affective disorders, especially depression and anxiety, as well as a high risk of suicidality and nonsuicidal self-injury

- Recent research trends include preliminary findings of elevated autistic traits in trans persons

- Gender-affirming surgery and/or cross-sex hormone treatment decrease mental health problems, body uneasiness, and gender dysphoria but not necessarily suicidality

- A high rate of trans persons find discrimination both in personal and professional lives

- Search for neurobiological correlates of transgenderism is still ongoing

- There have been promising functional MRI findings in voice perception and olfaction

\section{Key issues}

- Structural MRI research is often discrepant but consistently shows some effects

- Sample sizes are increasing in behavioral research but remain small in neurobiological studies

- Currently there is no neuro-affective research in trans persons

- There have been no randomized controlled trials yet on cross-sex hormone treatment

- There is a paucity of data in trans adolescents other three groups. Yet, at the brain level, group differences revealed a larger activation in cisgender boys relative to cisgender girls in the Rolandic operculum, and brain activity in the transgender groups was at levels in between these groups. In a small study of CSHT (six trans men and eight trans women), Sommer et al. (53) examined two languagebased tasks (verb generation and categorical decision) and reported an increase in language activation in both transgender groups after treatment, an effect that was associated with posttreatment estradiol levels for both sexes. Taken together, unfortunately, findings from this line of work examining sex-typical and sex-atypical neurocognitive differences are inconclusive because of very small samples $(51,53)$, effects that failed to reach statistical significance (52), lack of pretreatment data (50), or non-sex-specific hormonal effects (affecting both sexes) (53). While presenting an encouraging first set of preliminary studies to build upon, no conclusions can yet be drawn as to the effect of CSHT on sex-typical cognitive performance or the presence of pretreatment neurocognitive differences. Surprisingly, despite the high rates of affective disorders in trans persons, no published research to date has examined the neural correlates of emotional or affective responding in this population.

\section{LIMITATIONS AND FUTURE PERSPECTIVES}

Many studies in transgender persons suffer from methodological shortcomings, including small or very small sample sizes, ranging from 10 to 25 trans persons per group (with some exceptions, e.g., 48). Low statistical power is particularly problematic when the aim is to show the presence of an intermediate phenotype, which may make it difficult to show significant differences when results for the group of interest (trans persons) fall in between those of the control groups (cisgender persons). Thus, better-powered and multicenter studies are needed. Some initiatives in that direction have already formed, such as the European Network for the Investigation of Gender Incongruence (ENIGI initiative), which aims to assess differences in gender dysphoria among different European countries. Indeed, the ENIGI initiative revealed that some sociodemographic characteristics, including education level, age, and number of persons (trans men or trans women) presenting to the clinic, may already differ among European countries (Norway, Germany, Belgium, and the Netherlands) (54), suggesting the utility of including such factors in future work. Indeed, such countryspecific phenomena may account for some of the differences reported in the literature, for example, whether psychopathology is higher in trans men or trans women $(9,12,14)$. Even though such initiatives may need to overcome countryspecific limitations, including type of CSHT, legal struggles with acknowledgment of transition status, and recruitment pool (i.e., only through referral, mental health centers, or focus and activist groups), they allow sample sizes large enough to assess critical factors related to mental health and care, including quality of life and satisfaction before and after transition. Specifically, longitudinal research is urgently needed, given that treatment protocols and available care in specific countries have changed dramatically over the past few years. During such longitudinal work, the effects of CSHT on psychology during the transition process also require more attention. Likewise, neurobiological work would see significant improvements in explanatory power during joint initiatives or pooling of samples.

More comparative work between countries is also needed to establish optimal procedures, and the effects of CSHT on the brain and behavior require further confirmation and validation. Fortunately, the World Professional Association for Transgender Health has set out clearly defined treatment standards providing clinical guidance to meet the health care needs of trans persons that may be adjusted depending on the cultural context or the individual and that consequently provide a standardized yet sufficiently flexible framework (Standards of Care for the Health of Transsexual, Transgender, and Gender-Nonconforming People, Version 7) (2).

\section{CONCLUSIONS}

Since the beginning of the century, much progress has been made in charting the mental health needs of transgender 
persons alongside essential discoveries of underlying neurobiology $(33,37,38)$ and associated function $(47,48)$ (Figure 1). Available data suggest high rates of affective disorders $(9,17-19)$ that may decrease with treatment $(18,19)$, although suicide risk remains a cause for concern $(9,20,22$, 26). The currently increasing efforts to characterize the changes associated with transition to the preferred sex are promising $(19,41,45)$ but require larger-scaled collaborations for future validation and confirmation (54). We hope that such a research agenda will also contribute to a reduction in social stigma and an environment in which all persons receive optimal care regardless of sex or sexual orientation.

\section{AUTHOR AND ARTICLE INFORMATION}

From the Department of Experimental Clinical and Health Psychology, Ghent University; and the Department of Endocrinology and the Center for Sexology and Gender, Ghent University Hospital, Ghent, Belgium.

Address correspondence to Dr. Mueller (sven.mueller@ugent.be).

Dr. T'Sjoen has received grants (as principal investigator) from AstraZeneca, Bayer Schering, Ipsen, and Sandoz; consulting fees (as advisory board member) from Ipsen and Novartis; and speaking fees from Ferring and Novartis. The other authors report no financial relationships with commercial interests.

Received June 8, 2017; revision received Aug. 30, 2017; accepted Sept. 1, 2017; published online Oct. 20, 2017.

\section{REFERENCES}

1. American Psychiatric Association: Diagnostic and Statistical Manual of Mental Disorders, 5th ed: DSM-5. Washington, DC, American Psychiatric Association, 2013

2. Coleman E, Bockting W, Botzer M, et al: Standards of Care for the Health of Transsexual, Transgender, and Gender-Nonconforming People, Version 7. Int J Transgend 2012; 13:165-232

3. Arcelus J, Bouman WP, Van Den Noortgate W, et al: Systematic review and meta-analysis of prevalence studies in transsexualism. Eur Psychiatry 2015;30:807-815

4. Flores AR, Herman JL, Gates GJ, et al: How many adults identify as transgender in the United States? Los Angeles, UCLA, Williams Institute, June 2016

5. Jürgensen M, Kleinemeier E, Lux A, et al: Psychosexual development in adolescents and adults with disorders of sex development: results from the German Clinical Evaluation Study. J Sex Med 2013; 10:2703-2714

6. Mattila AK, Fagerholm R, Santtila P, et al: Gender identity and gender role orientation in female assigned patients with disorders of sex development. J Urol 2012; 188:1930-1934

7. Berenbaum SA, Beltz AM: Sexual differentiation of human behavior: effects of prenatal and pubertal organizational hormones. Front Neuroendocrinol 2011; 32:183-200

8. Zucker KJ, Bradley SJ, Oliver G, et al: Psychosexual development of women with congenital adrenal hyperplasia. Horm Behav 1996; 30: 300-318

9. Heylens G, Elaut E, Kreukels BPC, et al: Psychiatric characteristics in transsexual individuals: multicentre study in four European countries. Br J Psychiatry 2014; 204:151-156

10. Dhejne C, Lichtenstein P, Boman M, et al: Long-term follow-up of transsexual persons undergoing sex reassignment surgery: cohort study in Sweden. PLoS One 2011; 6:e16885

11. Claes L, Bouman WP, Witcomb G, et al: Non-suicidal self-injury in trans people: associations with psychological symptoms, victimization, interpersonal functioning, and perceived social support. J Sex Med 2015; 12:168-179
12. Gómez-Gil E, Trilla A, Salamero M, et al: Sociodemographic, clinical, and psychiatric characteristics of transsexuals from Spain. Arch Sex Behav 2009; 38:378-392

13. Hoshiai M, Matsumoto Y, Sato T, et al: Psychiatric comorbidity among patients with gender identity disorder. Psychiatry Clin Neurosci 2010; 64:514-519

14. Haraldsen IR, Dahl AA: Symptom profiles of gender dysphoric patients of transsexual type compared to patients with personality disorders and healthy adults. Acta Psychiatr Scand 2000; 102: 276-281

15. de Vries AL, Noens IL, Cohen-Kettenis PT, et al: Autism spectrum disorders in gender dysphoric children and adolescents. J Autism Dev Disord 2010; 40:930-936

16. Jones RM, Wheelwright S, Farrell K, et al: Brief report: female-tomale transsexual people and autistic traits. J Autism Dev Disord 2012; 42:301-306

17. Dhejne C, Van Vlerken R, Heylens G, et al: Mental health and gender dysphoria: a review of the literature. Int Rev Psychiatry 2016; 28: 44-57

18. Colizzi M, Costa R, Todarello O: Transsexual patients' psychiatric comorbidity and positive effect of cross-sex hormonal treatment on mental health: results from a longitudinal study. Psychoneuroendocrinology 2014; 39:65-73

19. Fisher AD, Castellini G, Ristori J, et al: Cross-sex hormone treatment and psychobiological changes in transsexual persons: two-year follow-up data. J Clin Endocrinol Metab 2016; 101:4260-4269

20. Clements-Nolle K, Marx R, Katz M: Attempted suicide among transgender persons: the influence of gender-based discrimination and victimization. J Homosex 2006; 51:53-69

21. Haas AP, Rodgers P, Herman JL: Suicide attempts among transgender and gender non-conforming adults: findings of the National Transgender Discrimination Survey. Los Angeles, UCLA, Williams Institute, Jan 2014

22. Reisner SL, White JM, Bradford JB, et al: Transgender health disparities: comparing full cohort and nested matched-pair study designs in a community health center. LGBT Health 2014; 1:177-184

23. Davey A, Arcelus J, Meyer C, et al: Self-injury among trans individuals and matched controls: prevalence and associated factors. Health Soc Care Community 2016; 24:485-494

24. Holt V, Skagerberg E, Dunsford M: Young people with features of gender dysphoria: demographics and associated difficulties. Clin Child Psychol Psychiatry 2016; 21:108-118

25. Asscheman H, Giltay EJ, Megens JA, et al: A long-term follow-up study of mortality in transsexuals receiving treatment with cross-sex hormones. Eur J Endocrinol 2011; 164:635-642

26. Haas AP, Eliason M, Mays VM, et al: Suicide and suicide risk in lesbian, gay, bisexual, and transgender populations: review and recommendations. J Homosex 2011; 58:10-51

27. European Union Agency for Fundamental Rights: EU LGBT Survey: European Union Lesbian, Gay, Bisexual And Transgender Survey. Vienna, European Union Agency for Fundamental Rights, 2014

28. Liu RT, Mustanski B: Suicidal ideation and self-harm in lesbian, gay, bisexual, and transgender youth. Am J Prev Med 2012; 42: 221-228

29. Smith YLS, van Goozen SHM, Kuiper AJ, et al: Transsexual subtypes: clinical and theoretical significance. Psychiatry Res 2005; 137: 151-160

30. Nieder TO, Herff M, Cerwenka S, et al: Age of onset and sexual orientation in transsexual males and females. J Sex Med 2011; 8: 783-791

31. Smith ES, Junger J, Derntl B, et al: The transsexual brain: a review of findings on the neural basis of transsexualism. Neurosci Biobehav Rev 2015; 59:251-266

32. Luders E, Sánchez FJ, Gaser C, et al: Regional gray matter variation in male-to-female transsexualism. Neuroimage 2009; 46:904-907

33. Savic I, Arver S: Sex dimorphism of the brain in male-to-female transsexuals. Cereb Cortex 2011; 21:2525-2533 
34. Emory LE, Williams DH, Cole CM, et al: Anatomic variation of the corpus callosum in persons with gender dysphoria. Arch Sex Behav 1991; 20:409-417

35. Mueller SC, Landre L, Wierckx K, et al: A structural MRI study in transgender persons on cross-sex hormone therapy. Neuroendocrinology 2017; 105:123-130

36. Yokota Y, Kawamura Y, Kameya Y: Callosal shapes at the midsagittal plane: MRI differences of normal males, normal females, and GID. Conf Proc IEEE Eng Med Biol Sci 2005; 3:3055-3058

37. Garcia-Falgueras A, Swaab DF: A sex difference in the hypothalamic uncinate nucleus: relationship to gender identity. Brain 2008; 131: 3132-3146

38. Kranz GS, Hahn A, Kaufmann U, et al: White matter microstructure in transsexuals and controls investigated by diffusion tensor imaging. J Neurosci 2014; 34:15466-15475

39. Rametti G, Carrillo B, Gómez-Gil E, et al: White matter microstructure in female to male transsexuals before cross-sex hormonal treatment: a diffusion tensor imaging study. J Psychiatr Res 2011; 45: 199-204

40. Rametti G, Carrillo B, Gómez-Gil E, et al: The microstructure of white matter in male to female transsexuals before cross-sex hormonal treatment: a DTI study. J Psychiatr Res 2011; 45:949-954

41. Hulshoff Pol HE, Cohen-Kettenis PT, Van Haren NEM, et al: Changing your sex changes your brain: influences of testosterone and estrogen on adult human brain structure. Eur J Endocrinol 2006; 155(suppl):S107-S114

42. Kim TH, Kim SK, Jeong GW: Cerebral gray matter volume variation in female-to-male transsexuals: a voxel-based morphometric study. Neuroreport 2015; 26:1119-1125

43. Zubiaurre-Elorza L, Junque C, Gómez-Gil E, et al: Effects of crosssex hormone treatment on cortical thickness in transsexual individuals. J Sex Med 2014; 11:1248-1261

44. Hahn A, Kranz GS, Küblböck M, et al: Structural connectivity networks of transgender people. Cereb Cortex 2015; 25:3527-3534
45. Mueller SC, Wierckx K, Jackson K, et al: Circulating androgens correlate with resting-state MRI in transgender men. Psychoneuroendocrinology 2016; 73:91-98

46. Zhou J-N, Hofman MA, Gooren LJ, et al: A sex difference in the human brain and its relation to transsexuality. Nature 1995; 378: 68-70

47. Berglund H, Lindström P, Dhejne-Helmy C, et al: Male-to-female transsexuals show sex-atypical hypothalamus activation when smelling odorous steroids. Cereb Cortex 2008; 18:1900-1908

48. Burke SM, Cohen-Kettenis PT, Veltman DJ, et al: Hypothalamic response to the chemo-signal androstadienone in gender dysphoric children and adolescents. Front Endocrinol (Lausanne) 2014; 5:60

49. Junger J, Habel U, Bröhr S, et al: More than just two sexes: the neural correlates of voice gender perception in gender dysphoria. PLoS One 2014; 9:e111672

50. Carrillo B, Gómez-Gil E, Rametti G, et al: Cortical activation during mental rotation in male-to-female and female-to-male transsexuals under hormonal treatment. Psychoneuroendocrinology 2010; 35: 1213-1222

51. Schöning S, Engelien A, Bauer C, et al: Neuroimaging differences in spatial cognition between men and male-to-female transsexuals before and during hormone therapy. J Sex Med 2010; 7:1858-1867

52. Soleman RS, Schagen SE, Veltman DJ, et al: Sex differences in verbal fluency during adolescence: a functional magnetic resonance imaging study in gender dysphoric and control boys and girls. J Sex Med 2013; 10:1969-1977

53. Sommer IE, Cohen-Kettenis PT, van Raalten T, et al: Effects of crosssex hormones on cerebral activation during language and mental rotation: an fMRI study in transsexuals. Eur Neuropsychopharmacol 2008; 18:215-221

54. Kreukels BP, Haraldsen IR, De Cuypere G, et al: A European Network for the Investigation of Gender Incongruence: the ENIGI initiative. Eur Psychiatry 2012; 27:445-450 